\title{
Tunneling Images of Germanium Surface Reconstructions and Phase Boundaries
}

\section{Citation}

Becker, R. S., J. A. Golovchenko, and B. S. Swartzentruber. 1985. Tunneling Images of

Germanium Surface Reconstructions and Phase Boundaries. Physical Review Letters 54, no. 25: 2678-2680. doi:10.1103/physrevlett.54.2678.

\section{Published Version}

doi:10.1103/PhysRevLett.54.2678

\section{Permanent link}

http://nrs.harvard.edu/urn-3:HUL.InstRepos:29407046

\section{Terms of Use}

This article was downloaded from Harvard University's DASH repository, and is made available under the terms and conditions applicable to Other Posted Material, as set forth at http:// nrs.harvard.edu/urn-3:HUL.InstRepos:dash.current.terms-of-use\#LAA

\section{Share Your Story}

The Harvard community has made this article openly available.

Please share how this access benefits you. Submit a story.

Accessibility 


\title{
Tunneling Images of Germanium Surface Reconstructions and Phase Boundaries
}

\author{
R. S. Becker, J. A. Golovchenko, and B. S. Swartzentruber \\ AT\&T Bell Laboratories, Murray Hill, New Jersey 07974 \\ (Received 2 April 1985)
}

\begin{abstract}
We have imaged with the tunneling microscope the microscopic surface topography of germanium layers grown by molecular-beam epitaxy on $\mathrm{Si}(111)-7 \times 7$. The surface structures corresponding to $\mathrm{Ge}(111)-c 2 \times 8$ and $\mathrm{Ge}(111)-7 \times 7$ are resolved. The Ge- $c 2 \times 8$ structure is shown to consist of a coherent combination of two simpler units both of which exist independently on the surface. The associated phase boundaries are illustrated. The Ge $7 \times 7$ structure is shown to be similar to that obtained for $\mathrm{Si}(111)$. Defects in the cell are also imaged.

PACS numbers: $68.20 .+\mathrm{t}, 61.70 . \mathrm{Ey}$
\end{abstract}

We report on the observation of spatial images of germanium surface reconstructions and phase boundaries, obtained with the tunneling microscope. The surfaces studied were of germanium grown epitaxially on silicon (111). With proper treatment, LEED studies $^{1}$ have shown these to yield a wide range of reconstructions normally observed on clean silicon or germanium surfaces. For example, the germanium overlayer will take the surface habit of silicon (111), i.e., the $7 \times 7$ reconstruction, for layers less than a few thousand angstroms thick after molecular-beamepitaxy preparation. ${ }^{2}$ This enables us to make a direct comparison of tunneling images with those obtained ${ }^{3,4}$ for $\mathrm{Si} 7 \times 7$ to see whether these two structures are identical. Thermal processing of this very same sample causes the LEED pattern to change from $7 \times 7$ to a centered $2 \times 8$ structure. This is the pattern observed on clean germanium (111) surfaces and we present the first images of this phase as well. We also observe a $2 \times 2$ reconstruction and subunits of a $c 4 \times 2$ cell in the tunneling images, which cannot be uniquely deduced from LEED patterns also containing $2 \times 8$ regions. This leads to a straightforward model for the $c 2 \times 8$ structure. Finally we present the first spatial observation of surface phase boundaries between regions.

The tunneling microscope used in these studies is similar to that described by Binnig et al. ${ }^{5}$ While a preliminary discussion of the instrument constructed at AT\&T Bell Laboratories has already been presented, ${ }^{4}$ and the details will be presented elsewhere, the basic and salient features are described here for completeness. The tunneling microscope consists of a piezoelectric tripod whose legs form an orthogonal coordinate system on which is mounted a tungsten probe tip. Application of 0 to $1000 \mathrm{~V}$ to the metallic plates evaporated on the piezoelectric legs allows the tip to be electrically positioned anywhere within a 1$\mu \mathrm{m}$ cube. This structure is mounted on a piezoelectric plate that is kinematically and capacitively clampable so that it can step the tripod-probe-tip structure in intervals of $1 \mu \mathrm{m}$ to $200 \AA$, over millimeter ranges. With the aid of this structure and the tripod the probe tip can be brought to a few angstroms of a sample sur- face without "touching" the region to be scanned. With a bias of the order of a volt, in the case of semiconductors, applied between tip and sample, a feedback system positions the tip so as to maintain constant tunnel current to the sample. For the data to be reported here this tunnel current was $0.8 \mathrm{nA}$ for a tip bias of 1.5 to $2.5 \mathrm{~V}$ negative. The tip is then raster scanned across the sample surface laterally while the feedback system maintains constant probe-tip-sample distance. The recorded images represent the changing absolute position as the tip follows the surface topography. Typical raster rates are $40 \AA / \mathrm{sec}$ and areas scanned are of the order of 100 by $100 \AA$. The tunneling microscope resides in a UHV chamber of base pressure $2 \times 10^{-10}$ Torr. Also present in the chamber are annealing, sputtering, and low-energy electrondiffraction apparatus.

The sample reported on here is a 500 - $\AA$-thick germanium film that had previously been grown at $550^{\circ} \mathrm{C}$ on a $\mathrm{Si}(111)-7 \times 7$ substrate by molecular-beam epitaxy. ${ }^{1}$ The substrate consists of a 0.02 -in.-thick 0.05 $\Omega-\mathrm{cm}$ boron-doped silicon wafer aligned to within 6 arc min of the $\langle 111\rangle$ surface direction. After the transfer of the sample through air to our vacuum system it is sputter cleaned with a dose of $4 \times 10^{16}$ $\mathrm{Ar}^{+} / \mathrm{cm}^{2}$ and then annealed at $500{ }^{\circ} \mathrm{C}$ for $15 \mathrm{~min}$. After this treatment a very sharp $7 \times 7$ LEED reconstruction was observed. This agrees with the reports in Refs. 1 and 2 for the identical treatment which also showed no surface silicon present as determined by Auger-electron spectroscopy.

Figure 1 shows the tunneling image of the $7 \times 7$ germanium surface. The image is represented on a greyscale plot photographed off a computer terminal screen. White regions are high and black low, the total range on the picture being $\sim 1.0 \AA$. Because of thermal drift and uncertaintities in piezocrystal calibration the distance scale must be deduced from the diffraction kinematics. The data should therefore be interpreted as being presented in an oblique coordinate system; the absolute unit-cell dimensions are indicated in the figure captions. Rather than applying an affine transformation to the raw data we suggest that the 


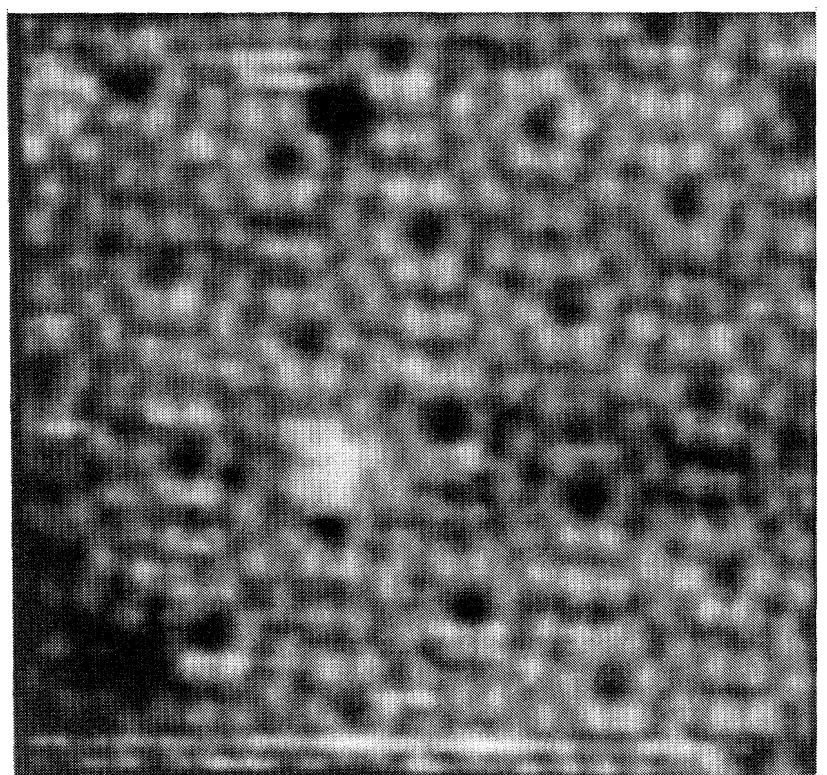

FIG. 1. Tunneling image of 500- $\AA$ Ge epitaxially grown on $\mathrm{Si}(111)$ showing $7 \times 7$ structure. The length of a unit-cell edge defined by the dark depressions is $28 \AA$ as calculated from electron diffraction.

viewer imagine that he is viewing the surface obliquely. The surface periodicity consists of rhombohedral unit cells with deep depressions at the corners and twelve protrusion inside. Thus the $\mathrm{Ge}(111)-7 \times 7$ structure appears quite similar to that first observed on silicon by Binnig et al. ${ }^{3}$ Point defects similar to those we have observed on silicon ${ }^{4}$ are also seen. A more careful inspection of the data suggests that the diameter of the deep corner depressions may be $10 \%$ to $20 \%$ smaller relative to the unit-cell size than on silicon. This observation must be interpreted with caution since we have no way of directly comparing the resolution in the germanium and silicon experiments.

The next step in sample preparation involved heating to $675^{\circ} \mathrm{C}$. This treatment results in an irreversible surface transformation to the centered $2 \times 8$ pattern as observed on our LEED apparatus. This again agrees with the prescription and results obtained in Refs. 1 and 2, where again no surface silicon is observed. The LEED pattern is identical to that obtained for bulk germanium (111) and corresponds to an unsolved surface structure.

The tunneling-microscope image corresponding to this state of the surface is shown in Fig. 2. This figure is remarkable in several ways. First, the lower lefthand region contains about a dozen unit cells corresponding to the $c 2 \times 8$ diffraction pattern. Figure 3 contains a sketch where the affine transformation is carried out and these unit cells are in the region labeled (a). The existence of a unit cell having this gen-

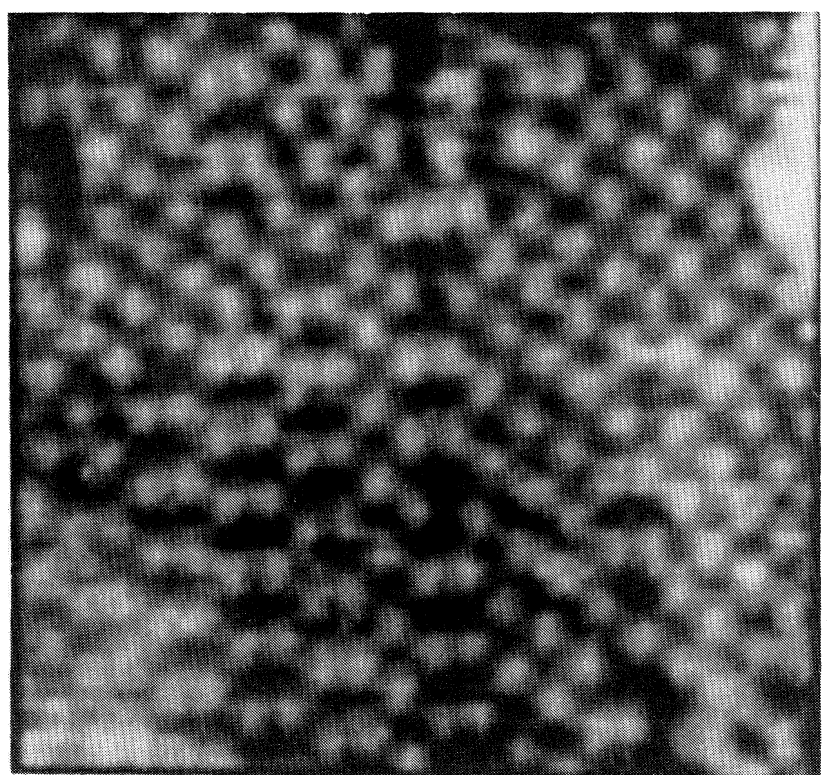

FIG. 2. Tunneling image of Ge $c 2 \times 8$ and $2 \times 2$ reconstructions.

eral symmetry is in agreement with the proposal of Chadi and Chiang ${ }^{6}$ and the more general theoretical analysis of missing LEED spots by Yang and Jona. ${ }^{7}$ The nature of the protrusions, as in the case of $\mathrm{Si}(111)-7 \times 7$ is not identified and may be either single atoms or bonded clusters. The upper right-hand side of Figs. 2 and 3 [in the region (c)] show what was at first for us an unexpected feature: a $2 \times 2$ surface reconstruction. This structure which we are unaware of having been reported before is in fact represented in the LEED pattern but since it shares the same halforder spots with the $c 2 \times 8$ structure, it has probably

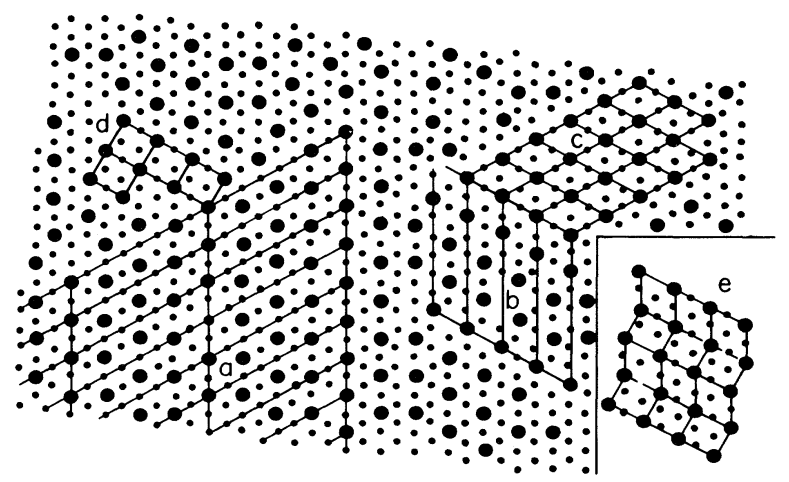

FIG. 3. Protrusions visible on Fig. 2 mapped to a hexagonal lattice. Unit cells of $c 2 \times 8$ are outlined in (a) and (b). Section (c) shows an extended region of $2 \times 2$, while (d) outlines some rectangular subunits of the $c 4 \times 2$ structure. The $c 2 \times 8$ pattern is reshown in (e) as an alternating pattern of $2 \times 2$ and $c 4 \times 2$ cells. 
gone unnoticed. This points to a major advantage of surface studies with the tunneling microscope. It seems clear to us that in many cases two or more surface phases may coexist on crystal surfaces, and standard methods of analysis like LEED, $x$-ray diffraction, ion scattering, etc., sampling large surface areas will fail to call attention to the fact. The tunneling microscope is particularly useful under these conditions.

The right-hand sides of Figs. 2 and 3 lin the region (b) ] also contain four unit cells of the $c 2 \times 8$ rotated $120^{\circ}$ from that in region (a). Finally, isolated sections of rectangular-looking cells which may be identified as subunits of a $c 4 \times 2$ structure are shown in the upper left-hand parts of Figs. 2 and 3 at (d).

Figure 3 clearly shows that the newly observed $2 \times 2$ and $c 4 \times 2$ cells appear to serve as the basic building blocks from which the $c 2 \times 8$ structure is built. This construction is indicated more clearly in Fig. 3(e) where the cells in (b) are shown to be a coherent ordering of the two smaller units. We note here that all the protrusions indicated in Fig. 3 can be interpreted to be centered on three-fold hollow sites above top-layer germanium (111)-plane atoms. The two kinds of building block cells may then not be too dissimilar to those illustrated by $\mathrm{Chadi}^{8}$ in his discussion of $7 \times 7$ and $5 \times 5$ structures.

The region bounding regions (b) and (c) in Fig. 3 corresponds to a distinct and simple phase boundary. That bounding regions (a) and (b) is a twin boundary. We believe this to be the first direct observation of such boundaries. The manner in which the crystal accommodates such transitions puts constraints on any underlying reconstruction which the tunneling images cannot record directly and may be a clue to that structure.

Aside from structural and geometrical questions it is interesting to speculate on the electrical behavior of the vacuum tunnel junction that that yields to topographs that we have presented. Similar topographs on metal samples require $\sim 10 \mathrm{mV}$ of bias to induce nonoampere tunnel currents. Here the entire applied voltage appears across the vacuum gap. We generally require about 100 times this bias with semiconductor surfaces to obtain good topographs. The explanation for this difference may be attributed to a large portion of the applied voltage appearing across space-charge regions inside the sample, and semiconductor material that may have been inadvertently transferred to the tip. Apparent height modulation also varies with applied bias with noticeable changes on $0.5-\mathrm{V}$ intervals. Without a knowledge of the true vacuum gap voltage or a general microscopic theory for the observed contrast it is difficult to assign absolute heights to surface features.

In conclusion, we have observed three new reconstructions with the tunneling microscope. The $\mathrm{Ge}(111)-7 \times 7$ structure appears quite similar to that for $\mathrm{Si}$. The $c 2 \times 8$ structure is observed and corresponds closely with the suggestions of Yang and Jona. A $2 \times 2$ reconstruction is also detected and the phase boundary between it and the $c 2 \times 8$ structure is resolved.

We wish to thank R. Malik for considerable assistance in teaching us the details of preparing this special sample. Discussions with E. G. McRae were invaluable in bringing us up to date on semiconductor surface reconstructions. Thanks are also due to $\mathrm{D}$. Hamann for valuable discussions and bringing our attention to the very interesting case of thick Ge epitaxial layers on $\mathrm{Si}$.

${ }^{1}$ H.-J. Gossman, J. C. Bean, L. C. Feldman, E. G. McRae, and I. K. Robinson, J. Vac. Sci. Technol. (to be published).

${ }^{2}$ E. G. McRae, H.-J. Gossman, L. C. Feldman, I. K. Robinson, and R. A. Malic, to be published.

${ }^{3}$ G. Binnig, H. Rohrer, Ch. Gerber, and E. Weibel, Phys. Rev. Lett. 50, 120 (1983).

${ }^{4}$ R. S. Becker, J. A. Golovchenko, and B. S. Swartzentruber, to be published.

${ }^{5}$ G. Binnig, H. Rohrer, Ch. Gerber, and E. Weibel, Phys. Rev. Lett. 49, 57 (1982).

${ }^{6}$ D. J. Chadi and C. Chiang, Phys. Rev. B 23, 1843 (1981).

${ }^{7}$ W. S. Yang and F. Jona, Phys. Rev. B 29, 899 (1984).

${ }^{8}$ D. J. Chadi, Phys. Rev. B 30, 4470 (1984). 


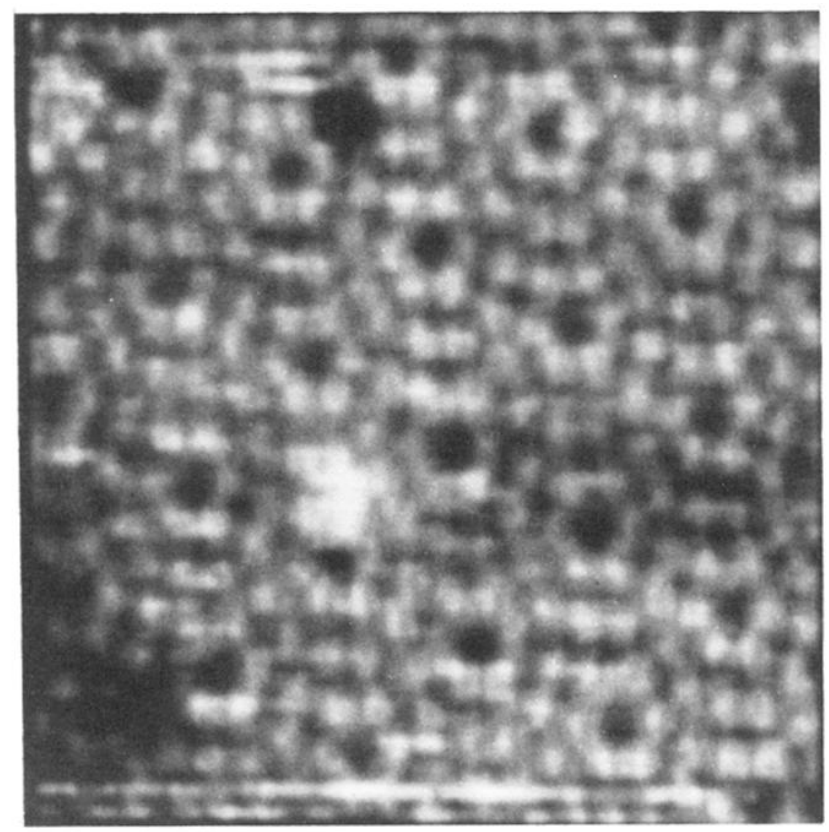

FIG. 1. Tunneling image of $500-\AA$ Ge epitaxially grown on $\mathrm{Si}(111)$ showing $7 \times 7$ structure. The length of a unit-cell edge defined by the dark depressions is $28 \AA$ as calculated from electron diffraction. 


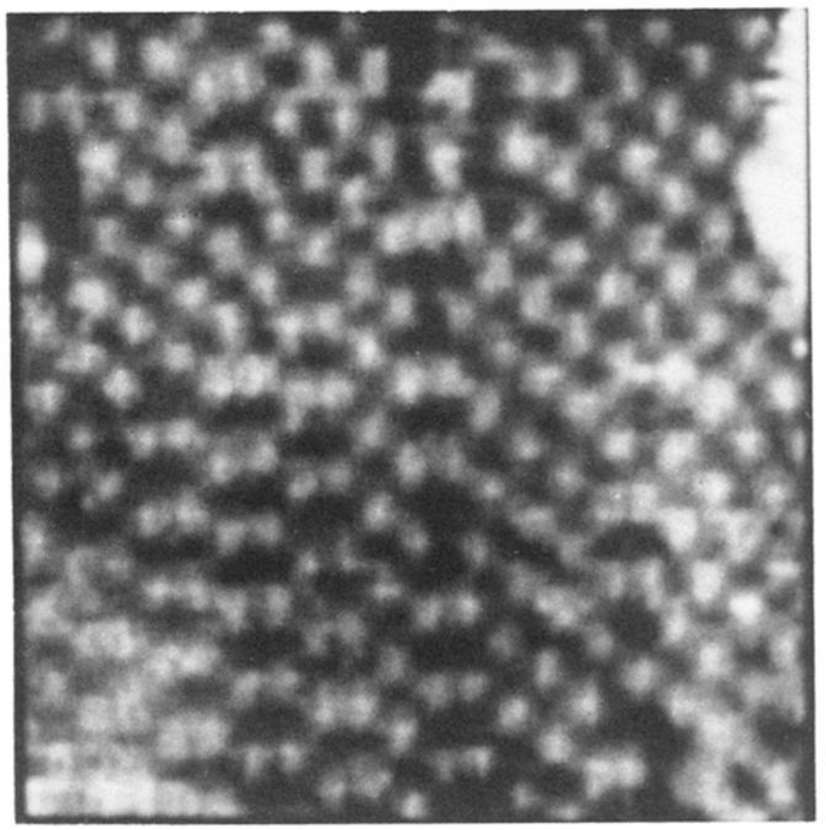

FIG. 2. Tunneling image of $\mathrm{Ge} c 2 \times 8$ and $2 \times 2$ reconstructions. 